\title{
NUMERICAL INVESTIGATION OF HEAT TRANSFER IN GARMENT AIR GAP
}

\author{
Yijie Zhang ${ }^{1,2}$, Juhong Jia ${ }^{3, *}$ \\ 1 School of Fashion Design and Engineering, Zhejiang Sci-Tech University, Hangzhou, Zhejiang 310018, China \\ 2 Shangyu College, Shaoxing University, Shaoxing, Zhejiang 312300, China \\ 3 School of Aerospace Engineering, Beijing Institute of Technology, Beijing 100081, China \\ ${ }^{*}$ Corresponding author. Email: jiajuhongit@126.com
}

\begin{abstract}
:
This article aimed to study the characteristics and mechanisms of $3 D$ heat transfer through clothing involving the air gap. A three-dimensional finite volume method is used to obtain the coupled conductive, convective, and radiative heat transfer in a body-air-cloth microclimate system. The flow contours and characteristics of temperature, heat flux, and velocity have been obtained. The reason for the high flux and temperature regions was analyzed. Computational results show that the coupled effect of the air gap and the airflow between the skin and garment strongly influences the temperature and heat flux distribution. There are several high-temperature regions on the clothing and high heat flux regions on the body skin because the conductive heat flux can cross through the narrow air gap and reach the cloth surface easily. The high-speed cooling airflow brings about high forced convective heat flux, which will result in the temperature increase on the upper cloth surface. The radiative heat flux has a strong correlation with the temperature gradient between the body and clothing. But its proportion in the total heat flux is relatively small.
\end{abstract}

\section{Keywords:}

Garment, air gap, heat transfer, conduction and convection, numerical simulation

\section{Introduction}

Thermal comfort is one of the important factors that meet customer's needs nowadays. The development of textile science and thermal control technology gives the chance to study the thermal comfort of clothing accurately. The garment system is constructed by clothing, air gap, and body skin [1]. Under ordinary temperature, thermal comfort can be achieved from the microclimate in this system with reasonable importation and interaction of conductive, convective radiative heat transfer, as shown in Figure $1\left(T_{\text {skin }}>T_{\text {cloth }}>0 \mathrm{~K}\right)$.

Some experimental studies are available in the literature where thermal comfort performance of 3D body was analyzed under different types of exposures [2, 3]. The bench top test was widely used to study heat transfer between the body and clothing either 2D or 3D [4-6]. However, the air gap was considered to be equally everywhere because the bench was considered to be flat [2, 7]. Santos [8] used a computational fluid dynamics (CFD) approach to perform numerical studies of fluid flow and heat transfer across cylindrical clothing microclimates with different values of microclimate thickness and argued that the increase of microclimate thickness can decrease the convective heat fluxes rapidly. Some scholars discussed a few finite volume method (FVM) models of heat transfer in the microclimate under the assumption of constant thermophysical properties of the fabric and constant convection in the air gaps

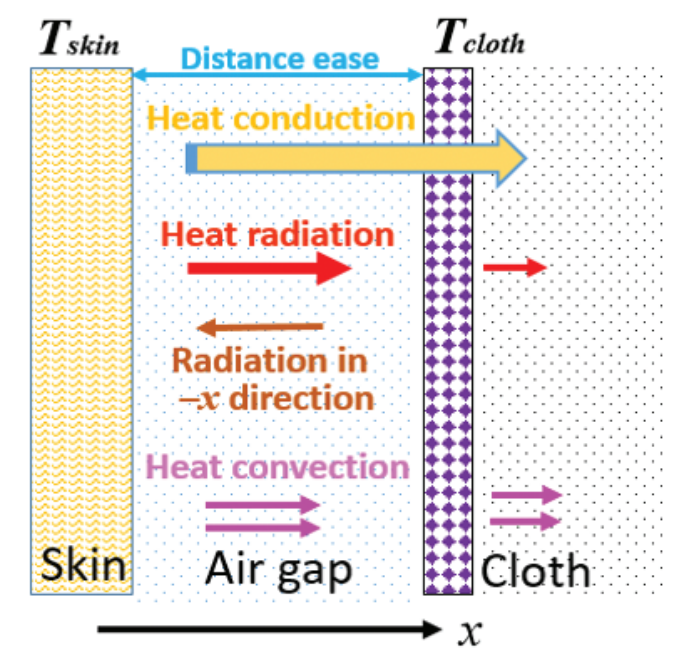

Figure 1. Schematic of heat transfer between the body skin and clothing.

[4, 6]. Ghazy [9] used the FVM method to solve the radiative transfer equations and energy equations and analyzed the effect of the dynamic air gap on heat transfer through the air gap. Udayraj [10] studied the heat transfer through air gaps by the CFD model, which considered conduction, convection, and radiation, and insisted that the heat flux was deviated to a lower level if neglect convection. 
As mentioned above, not enough work has been done on analyzing the heat transfer mechanism of the real 3D human body. The present study thus employs an FVM to develop a test method for determining the conduction, convection, and radiation heat flux between $3 \mathrm{D}$ body and clothing. The distributions of temperature on clothing surface and heat flux on the body surface are discussed. The relationship between heat flux and air gap distance, as well as airflow properties are analyzed.

\section{Physical problem}

\subsection{Physical model}

In the present work, heat transfer through clothing involving air gap is analyzed and temperature distribution on clothing surface is predicted. A standard female body and a type of fitting apparel are chosen here. The surface of the female body is established by the CLO 3D virtual try-on programs. Then, the girth feature curves were extracted by adding distance ease to the editable feature curves of the initial 3D mannequin [11]. Basic dimensions of body and clothing are given in Table 1.

\subsection{Mesh generation}

The unstructured grids are generated with fine mesh near walls by the commercial mesh generation software ICEM-CFD 17.0. For the symmetry of the human body, only half-geometry meshes are generated in this study to reduce computation load and improve precision. 3D garment and mesh are shown in Figure 2.

\subsection{Landmarks and ease allowance measurement}

This study focused on the distance ease in the horizontal direction, and four characteristic landmarks (Figure 3(a)) were selected to measure the distance ease and airflow contours. The virtual model of a clothed mannequin was simulated by commercial software Geomagic Qualify to obtain the 3D air gap distance, namely distance ease (Figure 3(b)). The distance ease is below $5 \mathrm{~mm}$ at the location of the shoulder, right side bust, side waist, and side thigh. The ease allowance value is between $5 \mathrm{~mm}$ and $15 \mathrm{~mm}$ at the chest, waist, and abdomen.

\section{Mathematical modeling and solution}

\section{procedure}

\subsection{Mathematical model}

Coupled Reynolds-averaged Navier-Stokes (RANS) equations [12] with radiative transfer equations (RTE) were

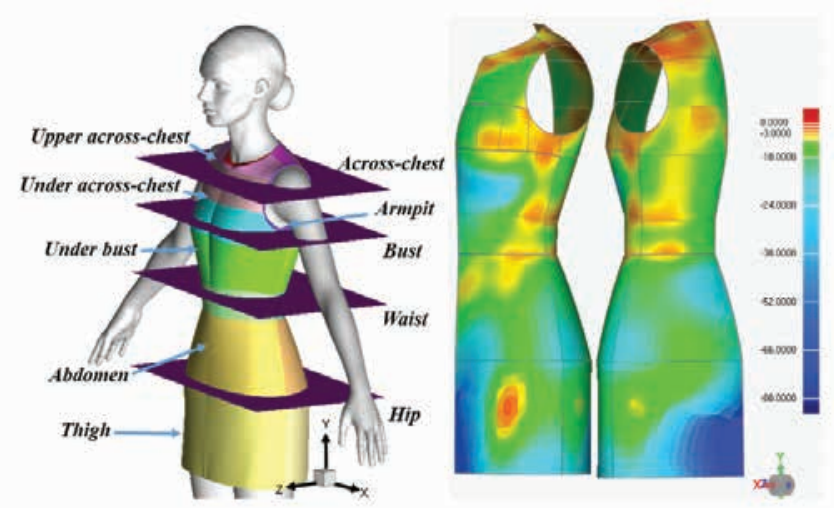

Figure 3. Landmarks and 3D distance ease: (a) landmarks and (b) distance ease.
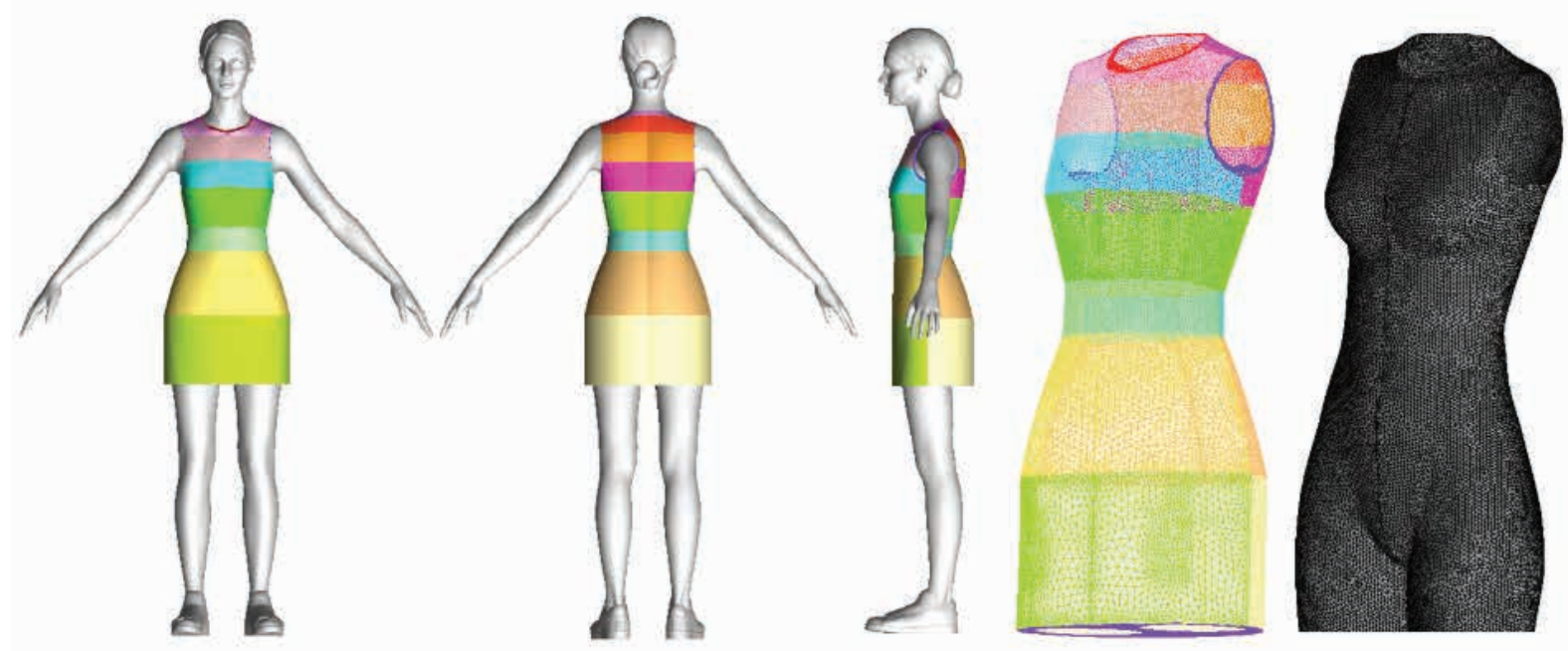

Figure 2. 3D garments and mesh domain.

Table 1. Basic dimensions of 3D mannequin and clothing

\begin{tabular}{|c|c|c|c|c|}
\hline & Bust girth $\mathbf{( c m )}$ & Waist girth $\mathbf{( c m )}$ & Hip girth (cm) & Garment length (cm) \\
\hline Human body & 84 & 66 & 90 & 80 \\
\hline Tight cloth & 88 & 68 & 96 & 80 \\
\hline
\end{tabular}


used to simulate heat transfer and fluid flow in a cloth-air-skin system. Three-dimensional continuity, momentum, and energy equations with the realizable $k-\varepsilon$ model $[13,14]$ in tensor forms were considered for modeling heat transfer and fluid flow in the air gap. The divergence of the radiative heat flux can be obtained using:

$$
\nabla \cdot q_{R}=\kappa\left(4 \pi I_{b}-G\right)
$$

where $I_{b}$ is the blackbody intensity, is defined as:

$$
I_{b}=\sigma T^{4} / \pi
$$

$G$ is the irradiation, is expressed as:

$$
G=\int_{\Omega=0}^{4 \pi} I(\Omega) d \Omega
$$

The solid angle $\Omega$ and radiation intensity I obtained by solving radiative transfer equations. The particles absorbing, emitting, and scattering are considered by the discrete ordinate radiation model (DORM).

The radiative transfer equations (RTE), used to calculate the radiation heat flux inside the air gap, are solved using the DORM, which is expressed as [15]:

$$
\nabla(I \hat{s})=\kappa \frac{\mathrm{n}^{2} \sigma T^{4}}{\pi}+I_{p}-\left(\kappa+\kappa_{p}+\gamma+\gamma_{p}\right) I(r, \hat{s})+\frac{\gamma}{4 \pi} \int_{\Omega=0}^{4 \pi} I\left(r, \hat{s}^{\prime}\right) \Phi\left(r, \hat{s}^{\prime}, \hat{s}\right) d \Omega
$$

\begin{tabular}{|c|c|c|c|}
\hline & Boundary types & Physical properties & Heat transfer \\
\hline Inlet & Uniform inlet flow & $\begin{array}{c}p_{\text {air }}=101325 p a \\
r_{\text {air }}=1.165 \mathrm{~kg} / \mathrm{m}^{3} \\
v_{\text {air }}=v y=0.5 \mathrm{~m} / \mathrm{s}\end{array}$ & $\begin{array}{c}T_{\text {air }}=283 \mathrm{~K} \\
C_{p_{-} \text {air }}=1.0 \mathrm{~kJ} / \mathrm{kg} \cdot \mathrm{K} \\
\mathcal{E}_{\text {air }}=0.02\end{array}$ \\
\hline Outlet & Back pressure outlet & $p_{\text {out }}=101325 \mathrm{pa}$ & - \\
\hline Body skin & No-slip, diffuse surfaces & $r_{\text {skin }}=860 \mathrm{~kg} / \mathrm{m}^{3}$ & $\begin{array}{c}T_{\text {skin }}=310 \mathrm{~K} \\
C_{p_{-} \text {skin }}=5.0 \mathrm{~kJ} / \mathrm{kg} \cdot \mathrm{K} \\
\mathcal{E}_{\text {skin }}=0.98\end{array}$ \\
\hline Cloth & Diffuse surfaces & - & $\varepsilon_{\text {cloth }}=0.95$ \\
\hline
\end{tabular}

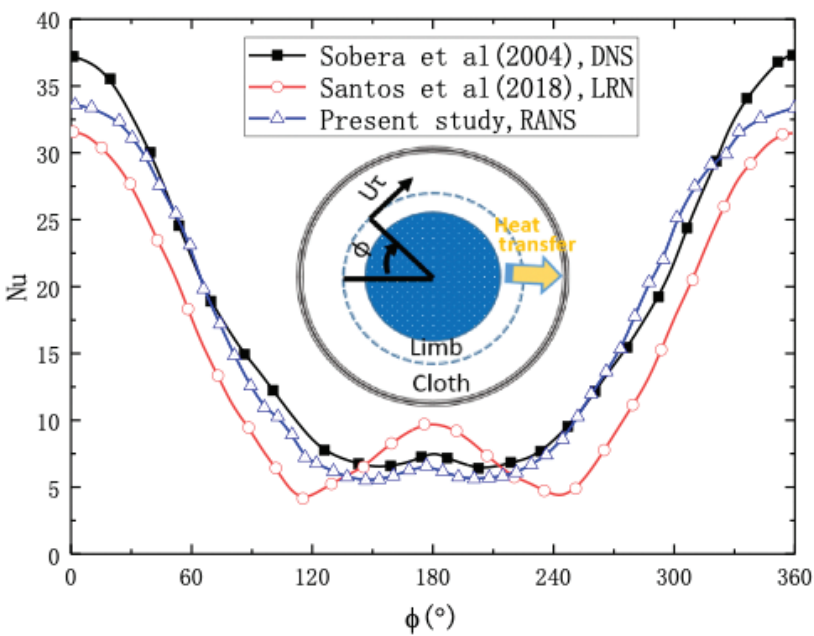

Figure 4. Natural convection and radiation in a square enclosure: (a) Nusselt number around skin and (b) time-averaged tangential velocity UT.

Table 2. Boundary conditions and physical properties

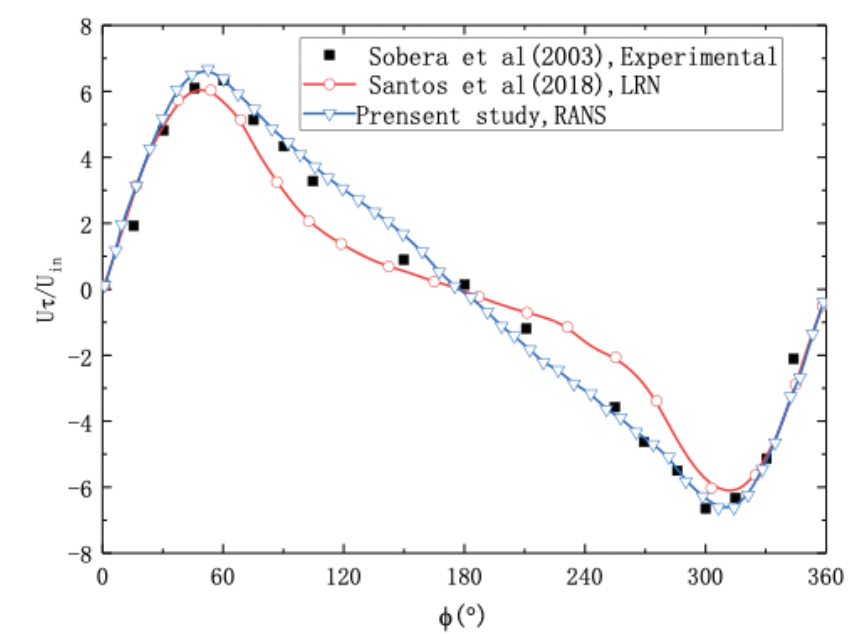

where $I_{p}$ is the equivalent emission of the particles, $\kappa_{p}, \gamma_{p}$ is the equivalent absorption coefficient and scattering coefficient. They can be expressed as:

$I_{\mathrm{p}}=\sum_{i=1}^{n} \varepsilon_{p i} A_{p i} \frac{\sigma T_{p i}^{4}}{\pi} N_{i}, \kappa_{\mathrm{p}}=\sum_{i=1}^{n} \varepsilon_{p i} A_{p i} N_{i}, \gamma_{p}=\sum_{i=1}^{n}\left(1-\alpha_{p i}\right)\left(1-\varepsilon_{p i}\right) A_{p i} N_{i}, A_{p i}=\frac{\pi d_{i}^{2}}{4}$

$N$. is the number density of the i particulate species, $\varepsilon_{p i}, T_{p i}, d_{i}, A_{p i}, \alpha_{p i}$ is its emissivity, temperature, diameter, projected area, and scattering factor. In this article, the radiation absorption coefficient is set to 0.0001 for air medium.

\subsection{Solution procedure}

To get the numerical solution for the heat transfer in air gap, some variable conditions, such as initial and boundary conditions, are needed. Owing to the complexity of heat transfer in air gap, some assumptions are adopted to simplify the numerical model. The flow inside air gap can be characterized as turbulent, weakly hem, natural convection due to buoyancy and heat radiation due to temperature gradient compressible flow containing forced convection due to the airflow from the clothing. The airflow within the fabric occurs in a laminar regime with low velocity. The buoyancy effects are considered because of natural convection heat transfer. The air bulk density is set 


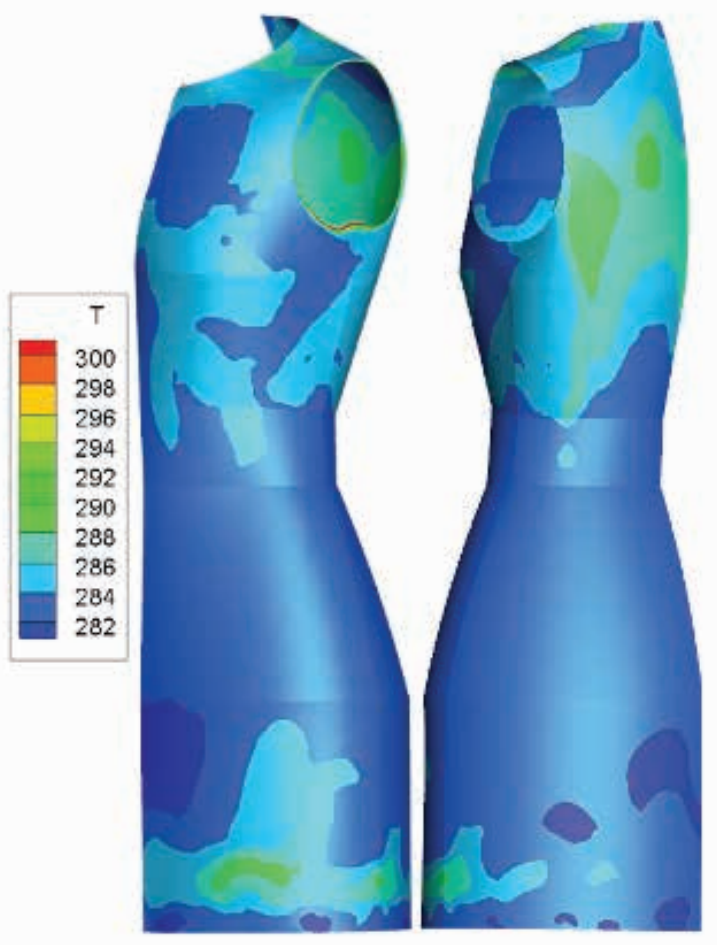

Figure 5. Temperature contours on the cloth surface.
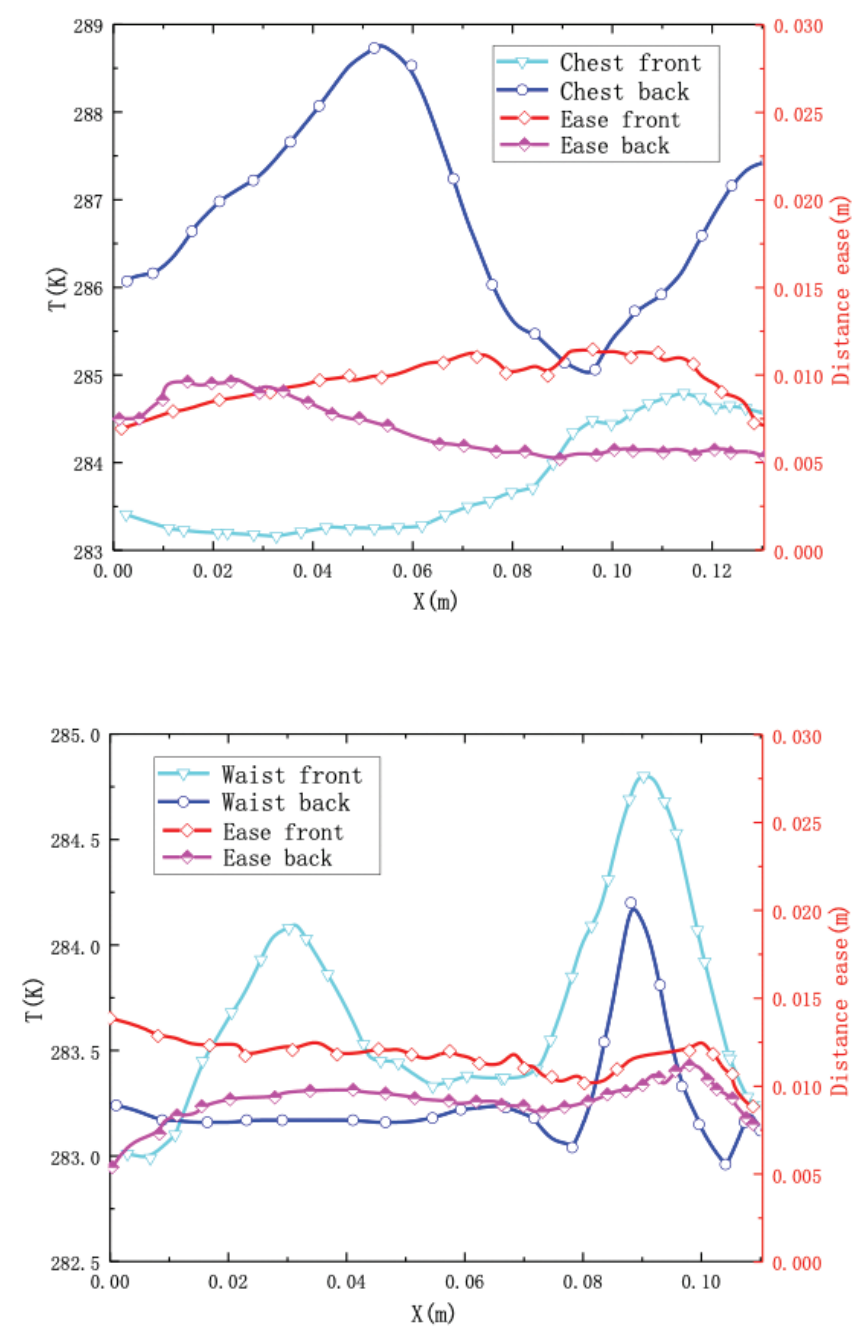

to $1.29 \mathrm{~kg} / \mathrm{m}^{3}$. Gravity is considered to be acting in a vertically downward direction. The gravitational acceleration vector is $-9.81 \mathrm{~m} / \mathrm{s}^{2}$. The mesh independence test was carried out with three different mesh scales, namely, 1.1 million for coarse mesh, 1.6 million for moderate, and 2.2 million for a refined mesh. The refined mesh was selected because of its accuracy to evolve the following calculations [16].

The freestream airflow was applied to the inlet, which is specified as a standard atmosphere with $T_{\text {air }}=283.15 \mathrm{~K}$ and $v_{\text {air }}=0.5 \mathrm{~m} / \mathrm{s}$, the gases emissivity is set to 0.02 . The outlet properties are extrapolated from inner cells. The body skin and inner fabric are considered as diffuse surfaces, with the emissivity of 0.98 and $0.95[8,17]$ ] (Table 2).

\section{Results and discussion}

\subsection{Validation of numerical methods}

The numerical method is validated first against the available results $[8,18,19]$ for a $2 \mathrm{D}$ horizontal impermeable cylinder, namely, human limb, with an external environment of $283 \mathrm{~K}$. The cylindrical clothing microclimates between the limb and the cloth surface are investigated. Figure $4(a)$ and (b) shows
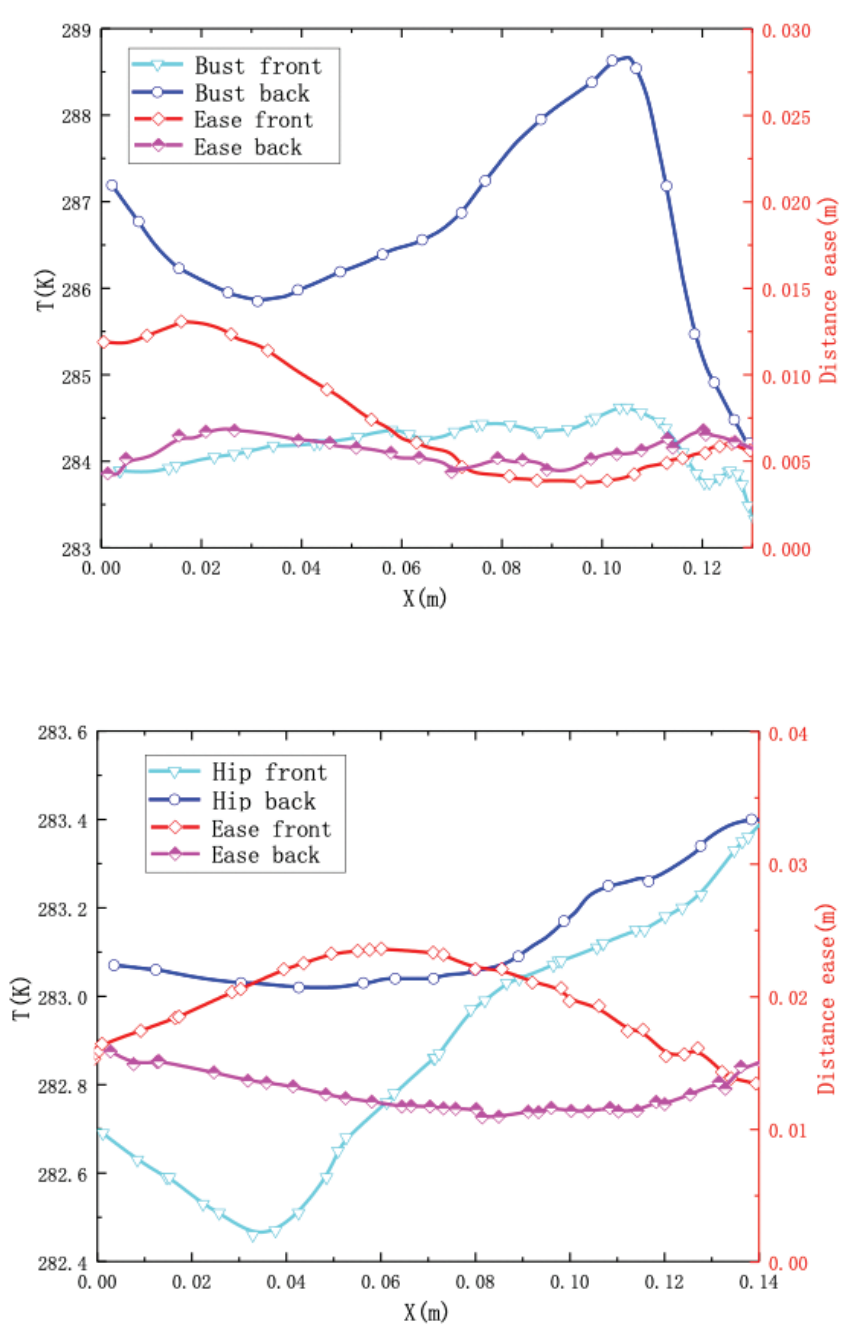

Figure 6. Temperature distributions on clothing surface cross-sections: (a) chest girth of clothing, (b) bust girth of clothing, (c) waist girth of clothing, and (d) hip girth of clothing. 
the present results for heat transfer Nusselt number and dimensionless tangential velocity variation along the skin, respectively, compared against the public works. The present method is found to be well in agreement with the available results and is satisfactory to carry out the following investigation.

\subsection{Temperature on the cloth surface}

The temperature contours under the condition of conduction, convection, and radiation are shown in Figure 5. As can be seen, the temperature distribution looks uneven all over the surface. The locations of the shoulder, upper chest, back chest, lower bust, and lower hem of cloth surface are the highest temperature regions.

To analyze the temperature quantitatively, temperature distributions near chest $(y=69 \mathrm{~cm})$, bust $(y=58 \mathrm{~cm})$, waist $(y=42 \mathrm{~cm})$, and hip girth $(y=22 \mathrm{~cm})$ are exhibited with the distance ease in Figure 6. As is shown, the temperature was increased with the ease allowance decrease. The temperature of the cloth was overall higher at the chest and bust girth (Figure 6(a) and (b)), and lower at the waist and hip (Figure $6(c)$ and (d)). This is partly because the conduction heat flux can cross through the narrow ease and reaches the cloth surface easily. However, the temperature increases nonlinearly with increasing ease distance. The most significant changes are noticeable between $X=0.05 \mathrm{~m}$ and $0.07 \mathrm{~m}$ at chest and between $X=0.09 \mathrm{~m}$ and $0.11 \mathrm{~m}$ at bust because of the high-

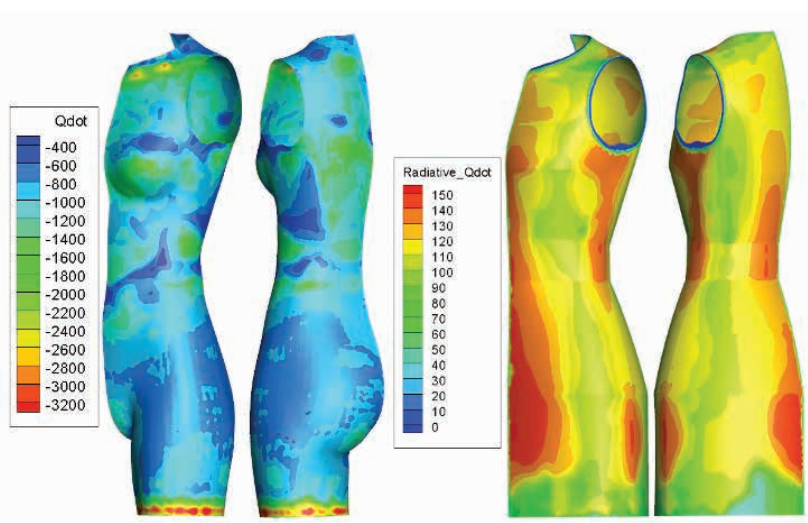

Figure 7. Heat flux contours distribution: (a) total heat flux on the body and $(\mathrm{b})$ radiative heat flux on clothing.

temperature results from high-speed cooling airflow, as we will explain in Chapter 4.4. This is a new and interesting observation of the present study.

\subsection{Heat flux on the body skin}

The total heat transfer and radiative heat flux are shown in Figure 7. Predictably, the air gap between the skin and the garment strongly influences the airflow and heat transfer in this region. The area around the neck, waist, and under the bust is the high heat flux regions (HFRs) where the lost total heat flux is above $1400 \mathrm{~W} / \mathrm{m}^{2}$ (Figure 7 (a)). This phenomenon mainly results from narrow distance ease. Interestingly, the total heat
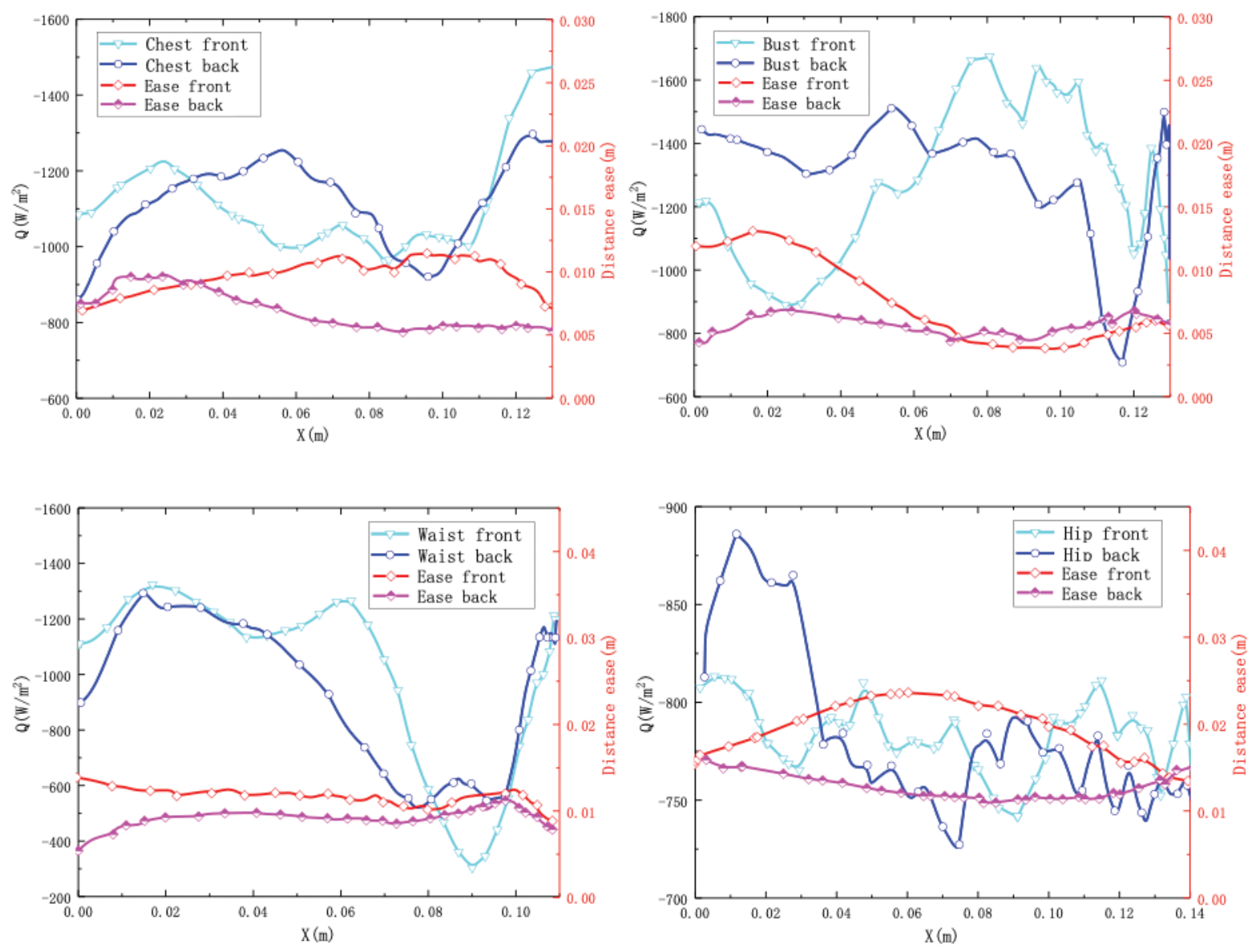

Figure 8. Heat flux on body surface cross-sections: (a) chest girth of cloth, (b) bust girth of cloth, (c) waist girth of cloth, and (d) hip girth of cloth. 
Table 3. High velocity and heat flux regions

\begin{tabular}{|c|c|c|c|c|}
\hline Location & HVR on cloth surface & Max Mach number & HFR on the body skin & Max heat flux $\left(\mathbf{W} / \mathbf{m}^{2}\right)$ \\
\hline neck & Front, side, clavicle & 0.020 & Front, side, clavicle & 2279.6 \\
\hline chest & Side & 0.016 & Middle, side & 1430.2 \\
\hline Bust & Middle, armpit & 0.019 & Middle, side & 1077.1 \\
\hline waist & Side, abdomen & 0.021 & Front, side & 1456.4 \\
\hline hip & Lateral & 0.004 & Front & 841.6 \\
\hline Thigh & Inner, lateral, round & 0.027 & Round & 3661.4 \\
\hline
\end{tabular}
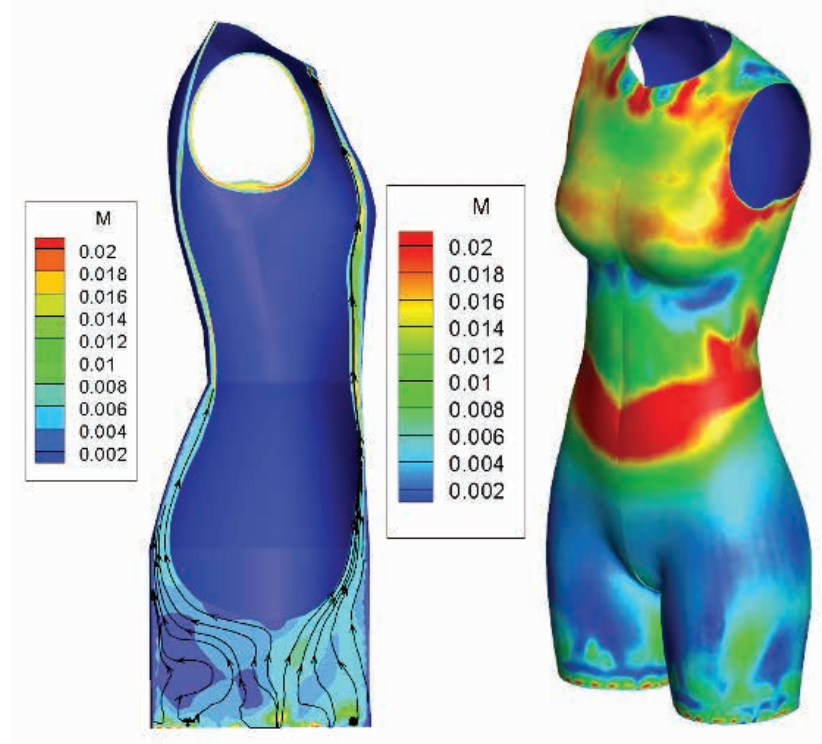

Figure 9. Airflow velocity in air gap: (a) velocity stream traces and (b) $3 \mathrm{D}$ velocity contours.

flux under the bust is also very high, for which the distance ease is wide enough. The circumference around the thigh has the maximum heat loss of more than $3200 \mathrm{~W} / \mathrm{m}^{2}$. These phenomena might result from air-forced convection, which is taken by the inflow of cooling air from the skirt's hemline. Compared with Figure $7(\mathrm{a})$, the high radiative HFR (Figure $7(b))$ is very different from that of total heat flux. The radiative heat flux on the cloth surface is relatively small about $150 \mathrm{~W} / \mathrm{m}^{2}$ on the area of middle bust, waist, and abandon. If considered Figure 6 and Figure $7(\mathrm{~b})$ together, we will find that the high cloth surface temperature regions in Figure 6 are similar to low radiative heat transfer regions in Figure $7(\mathrm{~b})$ to some extent. This is because the radiative exchange between the body and clothing depends on the distance between them and the temperature difference. The lower radiation heat transfer across air gap occurs due to the lower temperature gradient.

To analyze the heat transfer distribution quantitatively, heat flux curves at different cross-sections were obtained. It is to be noted that all the heat flux curves present wavelike appearance both on the front and backside of the body (Figure 8). The smaller distance ease leads to the higher heat flux on the whole, further confirming the influence of distance ease on the heat flux. It is also important to note here that the relationship between distance and heat flux is a little complex; the heat flux curve raises to a high level when the ease curve decreases especially at the location of $X=0.00 \mathrm{~m}$ to $0.02 \mathrm{~m}$ and $X=0.10 \mathrm{~m}$ to $0.12 \mathrm{~m}$. However, the heat flux curve does not keep pace with the distance ease curve (Figure 8(b) and (d)). Therefore, the total heat flux is affected by air gap distance, and the airflow between the body and clothing should be considered at the same time.

\subsection{Flow in the air gap}

To understand the physical reason behind heat flux and air gap width, another important factor of air velocity was considered. Based on airflow velocity obtained from the CFD analysis for the space of body and cloth, the stream traces are shown in Figure $9(a)$. As can be seen, the air velocity is relatively small between the double legs because of the wide space. The air velocity increases to a high level around the waist, bust, and chest because of narrow ease distance. The $3 \mathrm{D}$ velocity contours distribution between the body and clothing are shown in Figure 9(b). As shown, the high speed appears on the upper chest, bust, and waist with the Mach number of more than 0.02 .

If considered Figures 6 and 8 together, we will find that the peak value of temperature partly results from the high-speed cooling airflow. The comparison of high-velocity region (HVR) and HFR are shown in Table 3. These distributions are helpful to understand the influence of forced convection.

\section{Summary and conclusions}

This article aimed to investigate the 3D heat transfer of the bodyair-cloth system, coupled conduction, convection, and radiation models. The microclimate characteristics of temperature, heat flux, and velocity contours were discussed in the present work. The following are some of the major observations derived from the study:

1. The total heat flux is affected by several factors; the coupled effect of air gap and the airflow between the skin and garment strongly influence the temperature and heat flux distribution.

2. The temperature of the clothing was overall higher at the chest and bust girth due to the conduction heat flux, which can cross through the narrow ease and reach the cloth 
surface easily. The high-speed cool airflow brings about high forced convective heat transfer and then results in the temperature increase on the cloth surface.

3. The radiative heat flux is just a small part of total heat flux and has a strong correlation with the temperature gradient between the body and clothing.

\section{References}

[1] Kim, H., Kim, S. (2019). Heat storage and release characteristics of ceramic-imbedded woven fabric for emotional clothing. Autex Research Journal, 19(2), 165172.

[2] Su, Y., Li, R., Song, G., Li, J., Xiang, C. (2018). Modeling steam heat transfer in thermal protective clothing under hot steam exposure. International Journal of Heat and Mass Transfer, 120, 818-829.

[3] Fonrana, P., Saiani, F. (2018). Thermo-physiological impact of different firefighting protective clothing ensembles in a hot environment. Textile Research Journal, 88(7), 744753.

[4] Su, Y., He, J., Li, J. (2017). An improved model to analyze radiative heat transfer in flame resistant fabrics exposed to low-level radiation. Textile Research Journal, 87(16), 1953-1967.

[5] Bhattacharjee, D., Kothari, V. K. (2008). Prediction of thermal resistance of woven fabrics. Part II: Heat transfer in natural and forced convective environments, Journal of the Textile Institute, 99(5), 433-449.

[6] Sawcyn, C. M., Torvi, D. A. (2009). Improving heat transfer models of air gaps in bench top tests of thermal protective fabrics. Textile Research Journal, 79(7), 632-644.

[7] Udayraj, W. F. (2018). A three-dimensional conjugate heat transfer model for thermal protective clothing. International Journal of Thermal Sciences, 130, 28-46.

[8] Santos, M. S., Oliveira, D., Campos, J. B., Mayor, T. S. (2018). Numerical analysis of the flow and heat transfer in cylindrical clothing microclimates. Influence of the microclimate thickness ratio. International Journal of Heat \& Mass Transfer, 117, 71-79.
[9] Ghazy, A., Bergstrom, D. J. (2012). Numerical simulation of heat transfer in firefighters' protective clothing with multiple air gaps during flash fire exposure. Numerical Heat Transfer, Part A: Applications: An International Journal of Computation and Methodology, 61(8), 569-593.

[10] Udayraj, T. P., Das, A. (2017). Numerical modeling of heat transfer and fluid motion in air gap between clothing and human body: Effect of air gap orientation and body movement. International Journal of Heat and Mass Transfer, 108(3), 271-291.

[11] Li, J. T., Lu, G. D., Liu, Z. (2013). Feature curve-netbased three-dimensional garment customization. Textile Research Journal, 83(5), 519-531.

[12] Jia, J. H., Zhang, Y. J. (2020). Heat flux and pressure reduction using aerospike and counterfowing jet on complex hypersonic flow. International Journal of Aeronautical and Space Sciences, 21, 337-346.

[13] Jia, J. H., Fu, D. B., He, Z. P., Yang, J., Hu, L. (2020). Hypersonic aerodynamic interference investigation for a two-stage-to-orbit model. Acta Astronautica, 168:138-145.

[14] Metacomp Technologies Inc. (2013). CFD++ user manual. Agoura Hills, CA.

[15] Michael, F. M. (2003). Radiative heat transfer (2nd ed.). Academic Press (San Diego). pp. 55-73.

[16] Renato, M. C., Kleber, M. L., Marcos, F. C., Stavroula, B., Quaresma, J. N. N., et al. (2019). A review of hybrid integral transform solutions in fluid flow problems with heat or mass transfer and under Navier-Stokes equations formulation. Numerical Heat Transfer, Part B: Fundamentals, 4, 1-28.

[17] Watmough, D. J., Oliver, R. (1968). Emissivity of human skin in the waveband between 2 micra and 6 micra. Nature, 219(5154), 622-624.

[18] Sobera, M. P., Kleijn, C. R., Van den Akker, H. E. A., Brasser, $P$. (2003). Convective heat and mass transfer to a cylinder sheathed by a porous layer. AIChE Journal, 49, 3018-3028.

[19] Sobera, M. P., Kleijn, C. R., Van den Akker, H. E. A., Brasser, P. (2004). A multi-scale numerical study of the flow, heat, and mass transfer in protective clothing. Lecture Notes in Computer science, 3039, 637-644. 